\title{
Control in vivo de Moniliophthora roreri en Theobroma cacao, utilizando polisulfuro de calcio y silicosulfocálcico
}

\author{
In Vivo control of the Theobroma cacao pathogen \\ Moniliophthora roreri with calcium polysulfide and \\ calcium polysulfide added with ash
}

Fecha de recepción: 15 de febrero de 2017 Fecha de aprobación: 16 de junio de 2017
Lyda Esperanza Ochoa-Fonseca ${ }^{1}$ Sandra Isabel Ramírez-González²

Orlando López-Báez ${ }^{3}$

Saúl Espinosa-Zaragoza ${ }^{4}$ Álvaro Enrique Alvarado-Gaona ${ }^{5}$

Fernando Álvarez-Siman ${ }^{6}$

DOI: http://doi.org/10.19053/01228420.v14.n2.2017.7149

\section{Resumen}

Se evaluó en campo, bajo condiciones semicontroladas, la eficacia de los fungicidas minerales silicosulfocálcico (SSC) y polisulfuro de calcio (PC) para el control de la moniliasis [Moniliophthora roreri (Cif. \& Par.)] (Evans et al., 1978) del cacao, en el municipio de Tecpatán, Chiapas, México. Se inocularon frutos de 70 días de edad con el patógeno y se aplicaron los dos productos en concentración del $10 \%(\mathrm{v} / \mathrm{v})$ tres días antes o un día después de la inoculación. Las variables de estudio fueron: incidencia (\% I), índice de severidad externa (ISE) e índice de severidad interna (ISI), las dos últimas en escala de 0 a 5 . Los resultados indican que los dos productos, aplicados tres días antes o un día después de la inoculación con $M$. rore$r i$, inhibieron completamente el desarrollo de la enfermedad, ya que la incidencia, ISE e ISI presentaron valores de cero, mientras que en el tratamiento testigo (sin aspersión) estos valores fueron del $80 \%, 3.3$ y 3.8 , respectivamente. Se concluye que el SSC y el PC al $10 \%$ son una alternativa para el control de esta

1 M.Sc. Universidad Pedagógica y Tecnológica de Colombia (Tunja, Colombia).

2 Ph.D. Universidad Autónoma de Chiapas (Chiapas, México).

3 Ph.D. Universidad Autónoma de Chiapas (Chiapas, México).

4 Ph.D. Universidad Autónoma de Chiapas (Chiapas, México).

5 M.Sc. Universidad Pedagógica y Tecnológica de Colombia (Tunja, Colombia). alvaro.alvarado@uptc.edu.co. ORCID: 0000-0002-7024-5594.

6 Ph.D. Universidad Autónoma de Chiapas (Chiapas, México). 
enfermedad por su efectividad, bajo costo y facilidad de preparación; además de que la normatividad permite su aplicación en sistemas de producción orgánica.

Palabras clave: cacao; fungicidas minerales; índices de severidad.

\begin{abstract}
In the municipality of Tecpatán, Chiapas (Mexico), in a field trial was carried out under semicontrolled conditions, in order to evaluate the efficacy of mineral fungicides calcium polysulfide and calcium polysulfide added with ash to control the Theobroma cacao pathogen Moniliopthora roreri. Fruits of 70 days old were inoculated with the pathogen and the two products were applied in a concentration of $10 \%(\mathrm{v} / \mathrm{v})$ three days before or one day after inoculation. The variables evaluated were: incidence (\% I), external severity index (ISE) and internal severity index (ISI) (the last two on a scale of 0 to 5).

The results indicate that the two products applied three days before or one day after inoculation with $M$. roreri completely inhibited the development of the disease, due that the incidence, ISE and ISI presented values of zero, whereas in the control treatment (without sprinkling) these values were $80 \%, 3.3$ and 3.8, respectively. It is concluded that calcium polysulfide and calcium polysulfide added with ash in concentration of $10 \%$ are an alternative for controlling the disease due to their effectivness, low cost and ease of preparation. Moreover, the normativity allows their application in systems of organic production.
\end{abstract}

Keywords: cocoa; disease; mineral fungicides; severity index.

\title{
Como citar:
}

Ochoa-Fonseca LE., Ramírez-González SI., López-Báez O., Espinosa-Zaragoza S., Alvarado-Gaona AE., Álvarez-Siman F. Control in vivo de Moniliophthora roreri en Theobroma cacao, utilizando polisulfuro de calcio y silicosulfocálcico. Rev. Cien. Agri. 2017; 14(2): 59-66. 


\section{Introducción}

La producción de cacao (Theobroma cacao L.) en México se ha visto gravemente afectada desde la llegada de la moniliasis [Moniliophthora roreri (Cif. \& Par.)] al país, en el año 2005 (1, 2). Las pérdidas se estiman en más del $50 \%$ de la producción y ocasionan un fuerte impacto económico, social y ambiental en las regiones productoras de Tabasco y Chiapas $(1,3)$. Para el manejo de esta enfermedad, los productores realizan, básicamente, labores culturales como la poda de los árboles y la remoción de frutos enfermos, las cuales, si bien han demostrado ser eficaces en numerosos estudios $(4,5)$, no se implementan de forma rigurosa, factor que, sumado a otros, lleva a un bajo nivel de control. La aplicación de fungicidas no es una práctica ampliamente adoptada debido al alto costo de los productos o a su baja efectividad $(6,7)$.

Si bien la demanda de cacao orgánico muestra un incremento en los últimos años (8), los pequeños productores disponen de pocas herramientas para hacer frente a la moniliasis en sistemas de producción orgánica, así como en sistemas convencionales. Dentro de los productos estudiados para el control de este patógeno, y aceptados por las normas de producción orgánica, se encuentran los fungicidas cúpricos, como el óxido, hidróxido u oxicloruro de cobre $(9,10)$; sin embargo, las regulaciones cada vez son más restrictivas respecto al uso de este tipo de productos (Reglamento de Ejecución (UE) No. 354/2014). También se han adelantado estudios, principalmente en Costa Rica y México, con organismos antagonistas, como Trichoderma spp. (10, 11, 12, 13), y extractos vegetales $(14,15,16)$, pero no se encontraron reportes que indiquen su aplicación exitosa en cultivos a escala comercial.

Por otra parte, el uso de productos a base de sales minerales y de otros compuestos de origen natural ha mostrado buenos resultados en el control de diversos fitopatógenos; dos ejemplos conocidos son la aplicación de caldo bordelés para el control del mildeo (Plasmopara viticola) en vid (17) y de polisulfuro de calcio para el control de la roña (Venturiaina equalis) del manzano (18). En cacao, el cal- do bordelés se ha utilizado con éxito en el control de la pudrición negra, causada por Phytophthora palmivora $(19,20)$, y el polisulfuro de calcio alcanzó un alto nivel de control sobre $M$. roreri (21), tanto en condiciones in vitro como en plantación comercial.

Otros productos de este tipo: silicatos, fosfatos o bicarbonato de sodio, también han alcanzado buena efectividad para el control de enfermedades como mildeo (Bremia lactucae) en lechuga, mildeo polvoso (Blumeria graminis) en cebada o Phytophthora capsici en pimentón $(22,23,24)$. El interés en este tipo de productos deriva de su baja o nula toxicidad hacia mamíferos (incluidos los humanos) y hacia el medioambiente en general (25), sumado al hecho de que están permitidos por la normatividad de producción orgánica: Reglamento (CE) $n^{\circ}$ 834/2007, USDA-NOP, Codex Alimentarius para Alimentos producidos Orgánicamente $(26,27)$.

A partir de estas evidencias surgió el interés por determinar la eficacia de fungicidas minerales para el control de $M$. roreri. Con este fin se realizaron pruebas en condiciones in vitro, usando seis productos: caldo visosa, bicarbonato de sodio, caldo bordelés más permanganato de potasio, caldo bordelés más sulfato de magnesio y sulfato de zinc, polisulfuro de calcio y caldo silicosulfocálcico (28).

En una prueba de difusión en agar, el polisulfuro de calcio (PC) y el caldo silicosulfocálcico (SSC), en concentraciones de $10 \%$ a $50 \%$, inhibieron completamente el crecimiento micelial, la esporulación y la germinación de esporas del hongo, y en medio líquido (a concentración del $50 \%$ ) indujeron una mortalidad del $79.9 \%$ y $98.2 \%$ de las esporas, respectivamente, luego de $96 \mathrm{~h}$ de su exposición a estos productos. Los demás fungicidas minerales mostraron un efecto de inhibición menor; por tanto, los autores indican que los más promisorios para evaluación en condiciones de campo son los dos primeros. Con base en estos resultados, el presente estudio planteó el objetivo de establecer la eficacia del polisulfuro de calcio y el silicosulfocálcico para el control de M. roreri en condiciones semicontroladas en campo, las cuales se explican en el siguiente capítulo. 


\section{Materiales y métodos}

Para determinar, en un ensayo de campo, la eficacia de los dos fungicidas minerales, fueron asperjados en frutos inoculados manualmente con el patógeno. Esta metodología, además de garantizar un porcentaje alto de infección de los frutos, se aplicó con el objetivo de establecer si el PC y SSC tienen efecto preventivo o curativo; también permite determinar con mayor exactitud la eficacia de los productos, ya que se descartan factores como el lavado por las lluvias o un bajo cubrimiento de los frutos.

El ensayo se llevó a cabo en una plantación tradicional policlonal de cacao, de siete años de edad, ubicada en la rivera Menapac del municipio de Tecpatán (Chiapas, México), durante los meses de marzo a julio de 2014. En dicha plantación no se emplea ningún producto fitosanitario de síntesis química. Los frutos para realizar el ensayo se obtuvieron por polinización artificial y se cubrieron con bolsas de polietileno transparentes durante todo el periodo de estudio, con el fin de evitar una infección temprana con $M$. roreri, y según metodología descrita por Ramírez et al. (21).

El fungicida mineral PC se elaboró de acuerdo con el procedimiento descrito por Ramírez et al. (21); el SSC se elaboró de acuerdo con el procedimiento descrito por Restrepo (29), realizando la siguiente modificación en la composición: azufre $(5 \mathrm{~kg})$, óxido de calcio (10 kg), ceniza vegetal (5 kg), agua $(100$ L). A la edad de 70 días los frutos se inocularon con esporas de M. roreri, siguiendo la metodología descrita por Merchán (30), que consiste en depositar las esporas secas en un área de $4 \mathrm{~cm}^{2}$ en la parte ecuatorial del fruto, previamente humedecida con agua destilada. A fin de proporcionar una humedad óptima para la germinación de las esporas, durante dos días se dejó un algodón humedecido dentro de las bolsas que cubrían los frutos.

Para determinar si los productos tienen un efecto preventivo o curativo, los frutos del estudio se dividieron en dos grupos: el primero fue asperjado tres días antes de la inoculación (tratamiento preinoculación) y el segundo fue asperjado un día después de ésta (tratamiento posinoculación). Dadas las óptimas condiciones de humedad, se consideró que en 24 horas el hongo había realizado la penetración del fruto, pues en la literatura se reporta que ocho horas después de inoculación las hifas están creciendo intercelularmente en el parénquima (31). Los productos se aplicaron a cada fruto hasta punto de goteo, en concentración del $10 \%$ $(\mathrm{v} / \mathrm{v})$, con un atomizador manual.

Se utilizó un diseño experimental completamente al azar, con seis tratamientos y 15 repeticiones (frutos) por tratamiento: PC en preinoculación, PC en posinoculación, SSC en preinoculación, SSC en posinoculación, un testigo inoculado sin aspersión de productos y un testigo absoluto sin inoculación ni aspersión. Para obviar un posible efecto de variaciones en la resistencia genética de los clones hacia el patógeno, en cada árbol del ensayo se tuvo una repetición (fruto) para cada tratamiento. Las siguientes variables fueron cuantificadas en los frutos a la edad de 150 días: a) incidencia de $M$. roreri (\% I), indica el porcentaje de frutos que presentó algún síntoma o signo de la enfermedad; b) índice de severidad externa (ISE), indica el nivel de daño externo causado por la enfermedad, medido en una escala de cero a cinco (32), y c) índice de severidad interna, mide el nivel de daño interno (almendras) en los frutos, también en escala de cero a cinco (32). Para normalizar los datos del ISE e ISI, se transformaron mediante la fórmula [(valor $\left.+0.5)^{0.5}\right]^{*}$ 100. A los datos de las tres variables se les realizó análisis de varianza y comparación de medias a través de la prueba de Tukey. El análisis estadístico se llevó a cabo en el programa Statistical Analysis System (S.A.S) ${ }^{\circledR}$ versión 9.1 para Windows (SAS Institute Inc., Cary, NC).

\section{Resultados y discusión}

En la Tabla I se presentan los promedios de las tres variables cuantificadas en los frutos a la edad de 150 días, y en la Figura 1 se observa el aspecto de ellos. Los resultados de la variable incidencia de la enfermedad (\% I) muestran que los frutos asperjados con los dos fungicidas minerales, tanto antes como después de la inoculación con el hongo, no desarrollaron ningún síntoma o signo de moniliasis. De igual forma, en el testigo no inoculado (ab- 
soluto) los frutos permanecieron sanos, lo cual permite afirmar que el desarrollo de síntomas en otros tratamientos fue producto de la inoculación y no de la infección natural. Por el contrario, los frutos del testigo inoculado presentaron un alto porcentaje de incidencia, mostrando diferencia altamente significativa $(\mathrm{P}<0.05)$ con los demás tratamientos.

Tabla I. Incidencia (\% I), Índice de severidad externa (ISE) e Índice de severidad interna (ISI) en frutos de cacao asperjados con polisulfuro de calcio (PC) y silicosulfocálcico (SSC).

\begin{tabular}{|c|c|c|c|}
\hline Tratamiento & \% I & ISE & ISI \\
\hline PC preinoculación & $0^{\mathrm{b}}$ & $0^{\mathrm{b}}$ & $0^{\mathrm{b}}$ \\
\hline PC posinoculación & $0^{\mathrm{b}}$ & $0^{\mathrm{b}}$ & $0^{\mathrm{b}}$ \\
\hline SSC preinoculación & $0^{\mathrm{b}}$ & $0^{\mathrm{b}}$ & $0^{\mathrm{b}}$ \\
\hline SSC posinoculación & $0^{\mathrm{b}}$ & $0^{\mathrm{b}}$ & $0^{\mathrm{b}}$ \\
\hline Testigo no inoculado & $0^{\mathrm{b}}$ & $0^{\mathrm{b}}$ & $0^{\mathrm{b}}$ \\
\hline Testigo inoculado & $80^{\mathrm{a}}$ & $3.3^{\mathrm{a}}$ & $3.8^{\mathrm{a}}$ \\
\hline
\end{tabular}

Promedios con la misma letra en la misma columna no presentan diferencia estadísticamente significativa para la prueba de Tukey $(\mathrm{P} \leq 0.05)$.
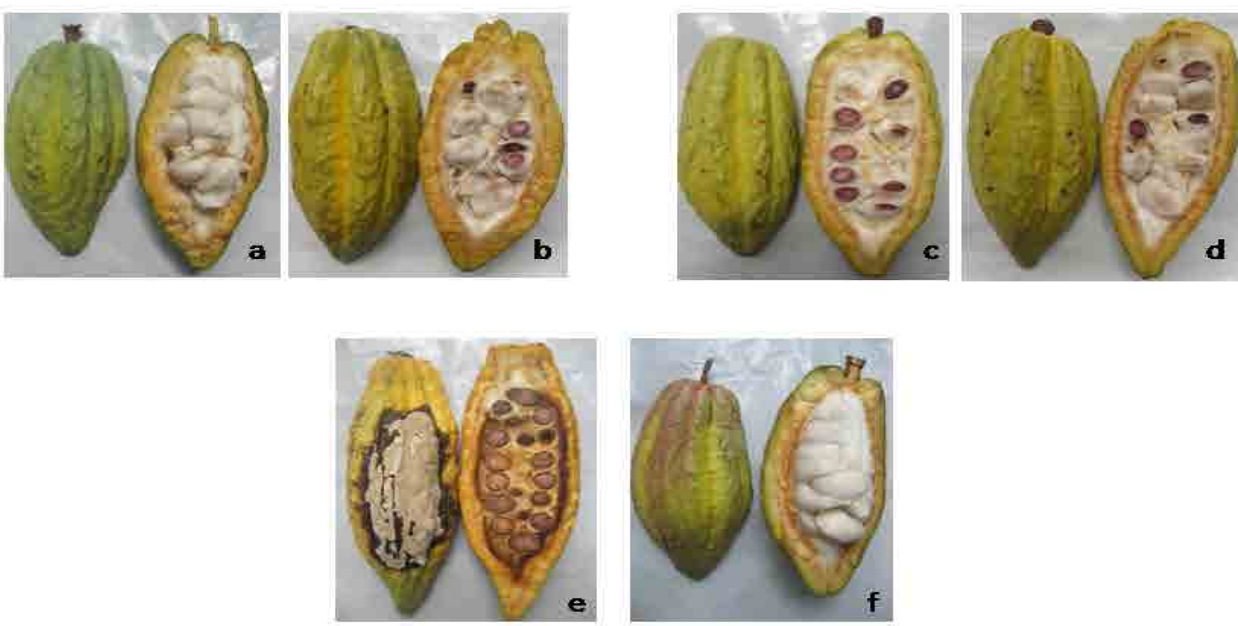

Fig. 1. Aspecto de los frutos al momento de la evaluación del ISE y el ISI. Tratamientos: a) PC preinoculación, b) PC posinoculación, c) SSC preinoculación, d) SSC posinoculación, e) testigo inoculado, f) testigo no inoculado (absoluto).

Los resultados obtenidos con el fungicida mineral PC, tanto en pre- como posinoculación, ratifican los hallazgos de Ramírez et al. (21), quienes aplicando esta misma metodología reportan que dicho producto inhibió completamente el desarrollo de la enfermedad, incluso con incidencia del 100\% en el testigo inoculado; adicionalmente, dichos autores corroboraron los resultados con una prueba a nivel de plantación comercial, en la cual los frutos tratados con PC presentaron $0.53 \%$ de incidencia, mientras que en el testigo sin aspersión fue del $69.6 \%$, y en un tratamiento con manejo cultural (remoción de frutos enfermos) fue de $21 \%$.

Dadas las diferencias metodológicas entre estudios orientados al control de la moniliasis, entre otros factores, no es posible realizar comparaciones directas frente a los resultados reportados por otros 
autores sobre el manejo de la enfermedad. Sin embargo, de manera general puede afirmarse que el control ejercido por el PC y el SSC supera los resultados obtenidos con otros productos estudiados o aplicados comercialmente, ya que se tienen los siguientes reportes que muestran que el hidróxido de cobre, producto permitido dentro de la normatividad de producción orgánica, y que ha sido evaluado en varios estudios realizados en Costa Rica, ha mostrado mayor eficacia que otros productos biológicos o de síntesis química, pero no ha logrado reducir la incidencia de moniliasis en más de un $20 \%$ frente a los tratamientos testigo.

Para ilustrar lo mencionado anteriormente, Hidalgo et al. (33) probaron la aplicación mensual de hidróxido de cobre y de dos asilados de Clonostachys rosea, encontrando el mejor control de M. roreri con el hidróxido de cobre, cuya incidencia fue de $60 \%$, apenas un $20 \%$ menor a la observada en el tratamiento testigo. Por su parte, los aislados de Clonostachys no lograron reducir la incidencia, aunque uno de ellos redujo el porcentaje de mazorcas esporuladas de $30 \%$ a $16 \%$. En otro estudio se evaluaron hidróxido de cobre, flutolanil, triadimenol, bitertranol y un aislado de Trichoderma asperellum; los resultados de los ensayos realizados durante tres años ratifican el hidróxido de cobre como el de mayor control, pero en ningún caso mostró una reducción de la incidencia mayor al $20 \%$. El flutolanil (300 g ingrediente activo. ha ${ }^{-1}$ ) le siguió en efectividad, y los demás tratamientos no mostraron un control significativo de la enfermedad.

En Ecuador se probó la rotación de fungicidas sistémicos y protectantes junto con la remoción quincenal de frutos enfermos, obteniendo el mejor resultado con la rotación Tega ${ }^{\circledR} 75-$ Antracol $^{\circ}$ 70-Silvacur ${ }^{\circledR} 300$-Antracol ${ }^{\circledR} 70$, cuya incidencia fue del $26.7 \%$ y una producción de $76.9 \%$ de cacao sano, frente al testigo, cuya incidencia fue del $49.7 \%$ y $57.2 \%$ de cacao sano. Es decir, la reducción de incidencia alcanzada por esta mezcla de tres fungicidas fue tan solo del $23 \%$, con un incremento de $19.7 \%$ en la producción de frutos sanos (34). Murillo y González (35) reportan una reducción considerable en la incidencia de moni- liasis en Costa Rica: de 93 \% (testigo) a $30.5 \%$, con la aplicación de clorotalonil, y a $42.2 \%$, con óxido cuproso, aun bajo condiciones de alta precipitación y en un cultivar susceptible al patógeno; sin embargo, puede ser un resultado inusual, pues otro estudio realizado con clorotalonil en la misma zona mostró una reducción de incidencia mucho menor: de 63 a $56 \%$ (36).

Como lo demuestran estos reportes, entre otros, con el uso de fungicidas no se ha logrado un control significativo y replicable de la enfermedad; adicionalmente, otros autores han demostrado las restricciones económicas de su aplicación, en especial cuando el precio del cacao es bajo o en plantaciones poco productivas $(36,37,34)$. Sin embargo, en el caso de extractos de plantas $(15,16,38)$ y en especial de los fungicidas minerales (21) PC y SSC, además de un buen control de la enfermedad, existen dos ventajas que los convierten en una mejor alternativa para los pequeños productores: su bajo costo, ya que pueden ser preparados por los mismos productores a partir de materiales de fácil consecución, y la posibilidad de ser aplicados en cultivos certificados orgánicamente. Teniendo en cuenta que el PC y el SSC ejercen un control similar de la enfermedad, el productor puede optar por la opción que le resulte más económica o de fácil preparación.

A partir de los resultados obtenidos en el presente estudio y de lo reportado por Ramírez et al. (21), se sugiere realizar pruebas sobre la frecuencia de aplicación del producto, tomando en cuenta la incidencia, la resistencia de los clones cultivados y las condiciones climáticas de las diferentes regiones productoras. Es importante aplicar estos fungicidas minerales en el marco de un sistema de manejo integrado de la moniliasis, en el cual se implementen prácticas culturales que mejoran la eficacia de control y optimizan los costos de producción.

\section{Conclusiones}

En condiciones semicontroladas en campo, los fungicidas minerales polisulfuro de calcio y silicosulfocálcico fueron una alternativa eficaz para controlar la moniliasis del cacao, pues inhibieron 
completamente el desarrollo del hongo al ser aplicados en concentración del 10\%, mientras que el tratamiento testigo presentó incidencia superior al $80 \%$.

Los dos fungicidas minerales mostraron acción preventiva y curativa, ya que controlaron la enfermedad al ser aplicados antes o después de la inoculación del patógeno. La acción preventiva se observó en aplicaciones hechas hasta 72 horas antes de la infección del fruto, y la acción curativa se observó hasta pasadas 24 horas después de la infección.

Estos fungicidas pueden ser aplicados en sistemas de producción orgánica o convencional, ya que están permitidos por la normatividad de producción orgánica; esto los hace productos versátiles que pueden ser incorporados en programas de manejo integrado de la moniliasis.

\section{Referencias}

(1) Ramírez S. La moniliasis, un desafío para lograr la sostenibilidad del sistema cacao en México. Tecnol Marcha. 2008; 21 (1): 97110.

(2) Díaz O., Aguilar J., Rendón R., Santoyo V. Current state of and perspectives on cocoa production in Mexico. Rev Cien e Inv Agraria. 2013; 40 (2): 279-289. DOI: http://doi.org/10.4067/ S0718-16202013000200004.

(3) Avendaño C., Villarreal J., Campos E., Gallardo R., Mendoza A., Aguirre J., Sandoval A., Espinoza S. Diagnóstico del cacao en México. Universidad Autónoma de Chapingo, SINAREFI, INIFAP. México. 2011.

(4) Soberanis W., Ríos R., Arévalo E., Zúñiga L., Cabezas O., Krauss $U$. Increased frequency of phytosanitary pod removal in cacao (Theobroma cacao) increases yield economically in eastern Peru. Crop Protect. 1999; 18 (10): 677-685. DOI: http://doi. org/10.1016/S0261-2194(99)00073-3.

(5) Evans H. Frosty Pod Rot (Moniliophthora roreri). En: Cacao Diseases: A History of Old Enemies and New Encounters. Bailey B, Meinhardt A, Lyndel W (Eds.) pp. 63-96. Springer. 2016. DOI: http://doi.org/10.1007/978-3-319-24789-2_3.

(6) Parra D., Sánchez L. El control de la moniliasis en el cacao. INIA Divulga. 2005; 6: 23-26.

(7) López O., González O., Lee V., Alvarado A., Ramírez S., Ramírez M., Méndez J., Gehrke M. Diagnóstico y técnicas para el manejo de la moniliasis del cacao. Universidad Autónoma de Chiapas, Universidad Pedagógica y Tecnológica de Colombia. 40 p. 2006.

(8) International Cocoa Organization -ICCO-. The Chocolate Industry. 2014. Disponible en: http://www.icco.org/about-cocoa/ chocolate-industry.html.
(9) Bateman R., Hidalgo E., García J., Arroyo C., Ten Hoopen G., Adonijah V., Krauss U. Application of chemical and biological agents for the management of frosty pod rot (Moniliophthora roreri) in Costa Rican cocoa (Theobroma cacao). Ann Appl Biol. 2005; 147: 129-138. DOI: http://doi.org/10.1111/j.17447348.2005.00012.x.

(10) Krauss U., Hidalgo E., Bateman R., Adonijah V., Arroyo C., Garcia J., Crozier J., Brown N., Ten Hoopen M., Holmes K. Improving the formulation and timing of application of endophytic biocontrol and chemical agents against frosty pod rot (Moniliophthora roreri) in cocoa (Theobroma cacao). Biol Control. 2010; 54: 230240. DOI: http://doi.org/10.1016/j.biocontrol.2010.05.011.

(11) Melnick R., Suárez C., Bailey B., Backman P. Isolation of endophytic endospore-forming bacteria from Theobroma cacao as potential biological control agents of cacao diseases. Biol Control. 2011; 57: 236-245. DOI: http://doi.org/10.1016/j.biocontrol.2011.03.005.

(12) Cuervo J., Sánchez V., Romero T., Ramírez M. Hypocrea/Trichoderma viridescens ITV43 with potential for biocontrol of Moniliophthora roreri Cif \& Par, Phytophthora megasperma and Phytophthora capsici. Afr J Mic Res. 2014; 8(16): 1704-1712. DOI: http://doi.org/10.5897/AJMR2013.6279.

(13) Crozier J., Arroyo C., Morales H., Melnick R., Strem L., Vinyard M., Collins R., Holmes K., Bailey B. The influence of formulation on Trichoderma biological activity and frosty pod rot management in Theobroma cacao. Plant Phathol. 2015; 64: 1385-1395. DOI: http://doi.org/10.1111/ppa.12383.

(14) Lozada B., Herrera L., Perea J., Stashenko E., Escobar P. Efecto in vitro de aceites esenciales de tres especies de Lippia sobre Moniliophthora roreri (Cif. y Par.) Evans et al., agente causante de la moniliasis del cacao (Theobroma cacao L.). Acta Agron. 2012; 61(2): 102-110. DOI: http://doi.org/10.15446/acag.

(15) Ramírez S., López O., Guzmán T., Munguía S., Espinosa S. Actividad antifúngica in vitro de extractos de Origanum vulgare L., Tradescantia spathacea Swartz y Zingiber officinale Roscoe sobre Moniliophthora roreri (Cif\& Par). Tecnol Marcha. 2011; 24 (2): 3-17.

(16) Ramírez S., López O., Guzmán T., Munguía S., Moreno J., Espinosa S. Biofungicidas de origen vegetal una alternativa para el manejo de la moniliasis del cacao en México. En: Memorias VII Congreso de la Red Latinoamericana de Ciencias Ambientales. San Carlos, Costa Rica; p. 46. 2013.

(17) Gessler C., Pertot I., Perazolli M. Plasmopara viticola: a review of knowledge on downy mildew of grapevine and effective disease management. Phytopathol Med. 2011; 50: 3-44. DOI: http://doi. org/10.14601/Phytopathol_Mediterr-9360.

(18) Holb I., Schnabel G. A detached fruit study on the post-inoculation activity of lime sulfur against brown rot of peach (Monilinia fructicola). Aust Plant Pathol. 2008; 37: 454-459. DOI: http:// doi.org/10.1071/AP08041.

(19) Hislop E. Studies on the chemical control of Phytophthora palmivora (Butl.) Butl. On Theobroma cacao L. in Nigeria. Ann Appl Biol. 1963; 52 (3): 465-480. DOI: http://doi.org/10.1111/j.1744-7348.1963.tb03770.x. 
(20) Adejumo T. Crop protection strategies for major diseases of cocoa, coffee and cashew in Nigeria. Afr J Biotechnol. 2005; 4 (2): 143-150.

(21) Ramírez S., López O., Guzmán T., Munguía S., Moreno J. El polisulfuro de calcio en el manejo de la moniliasis Moniliophthora roreri (Cif\& Par). Evans et al. del cacao Theobroma cacao L. Tecn en Marcha. 2011; 24 (4): 10-18.

(22) Dik A., Van der Gaag D., Van Slooten M. Efficacy of salts against fungal diseases in glasshouse crops. Comm Agric Appl Biol Sci. 2003; 68(4 Pt B): 475-485.

(23) Mitchell A., Walters D. Potassium phosphate induces systemic protection in barley to powdery mildew infection. Pest Manag Sci. 2004; 60(2): 126-134. DOI: 10.1002/ps.795

(24) French R., Avila F., Korndörfer G., Datnoff L. Silicon suppresses Phytophthora Blight development on bell pepper. J Phytopath. 2010; 158 (7-8): 554-560. DOI: http://doi.org/10.1111/j.14390434.2009.01665.x.

(25) Reuveni M., Reuveni R. Efficacy of foliar application of phosphates in controlling powdery mildew fungus on field-grown winegrapes: effects on cluster yield and peroxidase activity in berries. J Phytopath. 1995; 143: 21-25. DOI: http://doi.org/10.1111/j.1439-0434.1995.tb00194.x.

(26) Reglamento No. 834/2007 del Consejo de la Unión Europea, de 28 de junio de 2007, sobre producción y etiquetado de los productos ecológicos. Diario Oficial de la Unión Europea. p. L189/1-23. 2007. Disponible en: http://eur-lex.europa.eu/legal-content/ES/TXT/PDF/?uri = CELEX:32007R0834\&from $=$ ES.

(27) Codex Alimentarius para alimentos producidos orgánicamente. FAO-OMS. Tercera Edición. Roma. 52 p. 2007. Disponible en: ftp://ftp.fao.org/docrep/fao/010/a1385s/a1385s00.pdf.

(28) Ochoa L., Ramírez S., López O., Moreno J., Espinosa S. Efecto de preparados minerales sobre el crecimiento y desarrollo in vitro de Moniliophthora roreri (Cif. \& Par.) Evans et al. Rev Mex Cienc Agríc. 2015; 6(5): 1065-1075.

(29) Restrepo J. Manual Práctico El ABC de la agricultura orgánica y harina de rocas. Servicio de Información Mesoamericano sobre Agricultura Sostenible (SIMAS). Nicaragua. pp. 220. 2007.

(30) Merchán M. Avances en la investigación de la moniliasis del cacao en Colombia. En: La moniliasis del cacao. Enríquez, G. (Ed.). Informe Técnico No. 28. Centro Agronómico Tropical de Investigación y Enseñanza (CATIE), Costa Rica. pp. 53-69. 1980.

(31) Barros O. Investigaciones sobre el hongo Monilia roreri Cif and Par. causante de la pudrición acuosa de la mazorca del cacao; sus daños y su control. El Cacaotero Colombiano. 1977; 3: 4252.

(32) Sánchez J., Brenes O., Phillips W., Enríquez G. Metodología para la inoculación de mazorcas de cacao con el hongo Moniliophthora (Monilia) roreri. En: Proceedings of the Tenth International Cocoa Research Conference. Santo Domingo, República Dominicana. Cocoa Producers' Alliance. pp. 467-471. 1987.

(33) Hidalgo E., Bateman R., Krauss U., Hoopen M., Martinez A. A field investigation into delivery systems for agents to control Moniliophthora roreri. Europ J Plant Pathol. 2003; 109: 953-961. DOI: http://doi.org/10.1023/B:EJPP.0000003746.16934.e2.
(34) Ayala M., Navia D. Manejo integrado de moniliasis (Moniliophthora roreri) en el cultivo de cacao (Theobroma cacao L.) mediante el uso de fungicidas, combinado con labores culturales. CICYT-Escuela Superior Politécnica del Litoral. Ecuador. 6 p. 2008.

(35) Murillo D., González L. Evaluación en laboratorio y campo de fungicidas para el combate de la moniliasis del cacao. Agronomía Costarricense. 1984; 8(2): 83-89.

(36) González L., Sánchez A., Porras V., Umaña S., Murillo D. Evaluación del fungicida clorotalonil y de la destrucción de mazorcas enfermas en el combate de la moniliasis del cacao. Agronomía Costarricense. 1983; 7(1/2): 1-7.

(37) Sánchez L., Gamboa E., Rincón J. Control químico y cultural de la moniliasis (Moniliophthora roreri Cif \& Par) del cacao (Theobroma cacao L.) en el estado Barinas. Rev Fac Agron. 2003; 20: 188-194.

(38) Joya J., Ramírez S., López O., Alvarado A. Efecto antifúngico de hidrodestilados de Zingiber officinale Roscoe sobre Moniliophthora roreri (Cif \& Par). Rev Cienc Agric. 2015; 12(2): 21-29. DOI: http://doi.org/10.19053/01228420.4350. 